\title{
Physical characteristics at the turnover-points of relative biological effect (RBE) with linear energy transfer (LET)
}

To cite this article before publication: Bleddyn Jones et al 2019 Phys. Med. Biol. in press https://doi.org/10.1088/1361-6560/ab52a5

\section{Manuscript version: Accepted Manuscript}

Accepted Manuscript is "the version of the article accepted for publication including all changes made as a result of the peer review process, and which may also include the addition to the article by IOP Publishing of a header, an article ID, a cover sheet and/or an 'Accepted

Manuscript' watermark, but excluding any other editing, typesetting or other changes made by IOP Publishing and/or its licensors"

This Accepted Manuscript is $\odot 2019$ Institute of Physics and Engineering in Medicine.

During the embargo period (the 12 month period from the publication of the Version of Record of this article), the Accepted Manuscript is fully protected by copyright and cannot be reused or reposted elsewhere.

As the Version of Record of this article is going to be / has been published on a subscription basis, this Accepted Manuscript is available for reuse under a CC BY-NC-ND 3.0 licence after the 12 month embargo period.

After the embargo period, everyone is permitted to use copy and redistribute this article for non-commercial purposes only, provided that they adhere to all the terms of the licence https://creativecommons.org/licences/by-nc-nd/3.0

Although reasonable endeavours have been taken to obtain all necessary permissions from third parties to include their copyrighted content within this article, their full citation and copyright line may not be present in this Accepted Manuscript version. Before using any content from this article, please refer to the Version of Record on IOPscience once published for full citation and copyright details, as permissions will likely be required. All third party content is fully copyright protected, unless specifically stated otherwise in the figure caption in the Version of Record.

View the article online for updates and enhancements. 


\title{
Physical Characteristics at the Turnover-points of Relative Biological Effect (RBE) with Linear Energy Transfer (LET).
}

\author{
Authors \\ Bleddyn Jones ScD MD, \\ Mark A Hill MSc PhD. \\ Campus Research Building, Roosevelt Drive, Oxford, OX3 7DG, UK \\ Correspondence to Prof. Bleddyn Jones at above address, \\ e-mail: Bleddyn.Jones@ oncology.ox.ac.uk
}

Gray Laboratory, Oxford Institute for Radiation Oncology and Biology, University of Oxford, Old Road

Purpose: This paper considers the kinematic physical characteristics of ionic beams for maximum relative bio-effectiveness (RBE).

Methods: RBE studies, based on heterogenous cell survival studies at different laboratories and linear energy transfer (LET) conditions for proton, helium, carbon, neon and argon ions have been further analysed to determine the LET $_{U}$ values where RBE is maximal and the LETRBE relationship has a turnover point. The SRIM stopping power software and other classical equations are used to determine the particle velocities, kinetic energies and their effective ionic charges at $\mathrm{LET}_{\mathrm{U}}$.

Results: The estimated mean LET $_{U}$ values increase with atomic number $(Z)$. Each LET $_{U}$ has a unique relativistic velocity, $\beta=\mathrm{v} / \mathrm{c}$, the velocity $\mathrm{v}$ expressed as a fraction of the speed of light, c), and which is non-linearly proportional to Z. For ions helium and heavier ions, these velocities indicate that the effective charge $Z^{*}$ is around 0.99 of the full $\mathrm{Z}$ value at each $\mathrm{LET}_{\mathrm{U}}$, with remarkably stable velocities of 3-4 nm.fs ${ }^{-1}$ per nucleon, or around 6-8 nm.fs ${ }^{-1}$ per unit Z. For $\mathrm{Z}=1$, (protons and deuterium) some values fall outside these ranges but the result depends on the mix of proton and deuterium used in experiments. An alternative index of $\beta A / Z^{2}(A$ is the atomic mass number), suggests an average velocity of around $15 \mathrm{~nm} . \mathrm{fs}^{-1}$ for each particle at $\mathrm{LET}_{\mathrm{U}}$. These distances, traversed in the time of the radiochemical process initiation, are all within the dimensions of the nucleosome. Curve fitting of the data set provides a predictive equation for $\mathrm{LET}_{U}$ for any ion, as $\mathrm{LET}_{U}=30.4+108.4 / 0.61 \quad(1-\operatorname{Exp}[-0.61 \sqrt{ }(\mathrm{Z}-1)])$ when normalised to protons. These data can be extended to heavier ions such as silicon and Iron and give values that are consistent with experimental data. 
Conclusions: Each ion probably has a unique $\operatorname{LET}_{\mathrm{U}}$ value. Kinematic studies show maximum bio-effectiveness occurs at particle velocities where electron stripping remains at around $99 \%$ and where the velocity per nucleon is around 3-4 nm.fs ${ }^{-1}$.

Advances in Knowledge: This study enhances the limited prior knowledge about the physical conditions of particle beams that provide maximum bio-effectiveness, with applications in particle radiotherapy, radiation protection and space travel.

\section{Introduction}

Early investigators into the bio-effectiveness of different radiation qualities discovered the phenomenon that increasing linear energy transfer (LET) initially caused apparent linear increments in RBE (relative biological effectiveness) for a variety of ions compared to a reference low LET radiation, followed by progressive reductions in RBE at higher LET values, often interpreted as an 'overkill effect' [1a-5], probably due to the concept of 'wasted dose' in a relevant biophysical volume sufficient to cause cell death. The position of maximum or ultimate bio-effectiveness, represented by the turnover-point of the LET-RBE relationship (where the LET value is defined as LET $_{U}$ ), has received relatively scant attention, especially as many authors had assumed, not unreasonably, that all energetic ions shared a common LET $_{U}[6-8 \mathrm{a}]$ of around 100-120 keV. $\mu \mathrm{m}^{-1}$. Data mining exercises and analysis in the literature also gave the same overall impression [9, 10]. Closer inspection suggests that individual ion species might have specific LET-RBE turnover points which increase with $\mathrm{Z}$ (the nuclear charge), leading to predictive RBE modelling based on the concept that each ion has an unique LET $_{U}$ value based on its $Z$ number [11-14].

Although photons ( $\gamma$-rays or $x$-rays) interact with matter by the formation of intermediary electrons, there are important kinematic differences. The former, whatever their energy, always travel at the speed of light $(c)$, and react with other atoms by means of electromagnetic fields; charged particles have velocities smaller than $c$, which are proportional to their kinetic energy and interact with other atoms mainly by electrostatic fields, which become more apparent to other atoms when the particles slow down, causing increased LET. 
- It is important to understand that LET is estimated within a series of equations developed by Bethe containing the expression $Z^{2} / \beta^{2}$ and $\beta$ separately, where $\beta$ is the velocity relative to the speed of light (c), so that LET increases with not only with Z but also with reductions in $\beta$ due to slowing with increasing distance in matter, leading to the formation of the Bragg peak.

These concepts are further investigated in this article by using rational approaches to finding Z-specific LET $_{U}$ values from biological data sets, analysing and investigating these with respect to particle velocities, 'effective Z values' which influence LET due to fully ionised atoms gaining electrons during their trajectories through matter or tissue. The particle velocities are further analysed in terms of distance (in nanometres) traversed per femtosecond, since these parameters respectively represent the nucleosome dimensions and the time in which the initial stages of radiochemistry operate, It is important to determine if the widely dispersed $\beta$ values for different ions at LET $_{U}$ can converge into a narrower band of values which incorporate relevant spatio-temporal considerations, in order to improve our overall understanding and to better inform the mathematical modelling of charged particle bio-effectiveness.

\section{Methods}

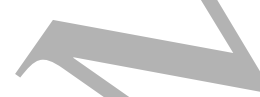

In previous studies [11-14], the LET $_{U}$ had been determined from the published LET values by assuming that LET $_{U}$ would be symmetrically placed between the maximum and the second highest RBE output results. In the present study, least squares fitting has been used to determine the intersection point between

(a) the linear function that governs the increment in RBE (and the increase in $\alpha$ and $\beta$ radiosensitivities) with LET, and

(b) the reciprocal curved function that governs the reduction in RBE (and the reduction of $\alpha$ and $\beta$ radio-sensitivities) with $L E T$,

These are simplifications of the equations given elsewhere [11] where further details of the $L E T_{U}$ based model are provided: the model effectively scales these radio-sensitivities to their ultimate (or maximum possible) values at $L E T_{U}$, but at larger $L E T$ values there is excess energy deposition which regarded as inefficient (or 'wasted dose') which results in a diminution of radiosenstivities and RBE. No assumptions are made as to whether cells are 
'hit' or not in a therapeutic setting. Some further details of the associated mathematics are given in the Appendix. The $\mathrm{LET}_{U}$ mean and $95 \%$ confidence limits were also estimated from the intersection points of the two functions, including their own $95 \%$ limits, accepting the smaller of the two which included most data points. The selected data sets were contained in the PIDE data base [9], and include the works of Belli et al (protons) [15], Barendsen (helium ions) [2], Weyrather et al (carbon ions) [5], Furusawa et al (neon ions) [4] and Blakely et al (argon ions) [3], but only included those which contained a reasonable number of data points, and where a maximum RBE region was apparent. No other ion species could be estimated due to the inadequacy of the data for this purpose. For the above ions, LET $\mathrm{U}$ was estimated for each level of cell survival fraction given in the data sets. Argon was added to the previously published analysis that included protons, helium, carbon, and neon ions [11]. Other ions were not included in the present analysis due to the limited number of data points, or if a clear turnover point was not apparent. Even so, some for these data sets included contain relatively few data points, so that the slopes of the upward and downward components of the overall relationship cannot be known to high precision. The estimated results and confidence limits must be regarded with caution due to such statistical limitations due to an imbalance of data points on the ascending and descending portions of the overall relationship.

Standard physical equations incorporated in the SRIM stopping power software [16] were used to estimate particle velocity $\beta$ (where $\beta=v / c$, the velocity divided by the speed of light) in water from the experimental LETU estimates obtained. The gain of electrons to fully electron-stripped ions results in a reduction of their effective charge $\left(\mathrm{Z}^{*}\right)$ or $\mathrm{Z}_{\text {eff. }}$ For ions with $Z=2$ and above, the Barkas equation [17] allows estimation of $Z_{\text {eff }}$ from $\beta$. Some of these formulations are given in the appendix for the benefit of biologists and physicians who may be involved in future experiments. The Katz ratio, $\mathrm{Z}_{\mathrm{eff}}{ }^{2} / \beta^{2}$, which is proportional to the radial dimension of secondary delta rays around each particle track, is also estimated at LET $\mathrm{U}$.

The interesting data of Todd [18], who used an almost constant kinetic energy per nucleon in nine different ions is re-analysed with respect to particle velocities and compared with the velocities associated with $\mathrm{LET}_{\mathrm{U}}$ in the present data set.

The data of Tsuruoka et al [19] is used to demonstrate the robustness of the model in predicting $\mathrm{LET}_{\mathrm{U}}$ values for silicon and iron ions that are compatible with each data set, which cannot individually be used to provide an estimate for $\mathrm{LET}_{\mathrm{U}}$. 
Mathematica (Wolfram, USA) software was used to fit published ion beam data sets obtained from the PIDE data base, and for all calculations and graphics.

\section{Results.}

The estimated mean $\mathrm{LET}_{U}$ values for five ion species are displayed in figure 1 , with their $95 \%$ confidence limits. The wide $95 \%$ confidence limits are due to the limited number of data points most of the LET-RBE experiments, the use of different cell lines with different RBE values, and experimental set-up variations in different laboratories. This degree of dispersion must be noted and further calculations below will use only the point estimates of the mean values.

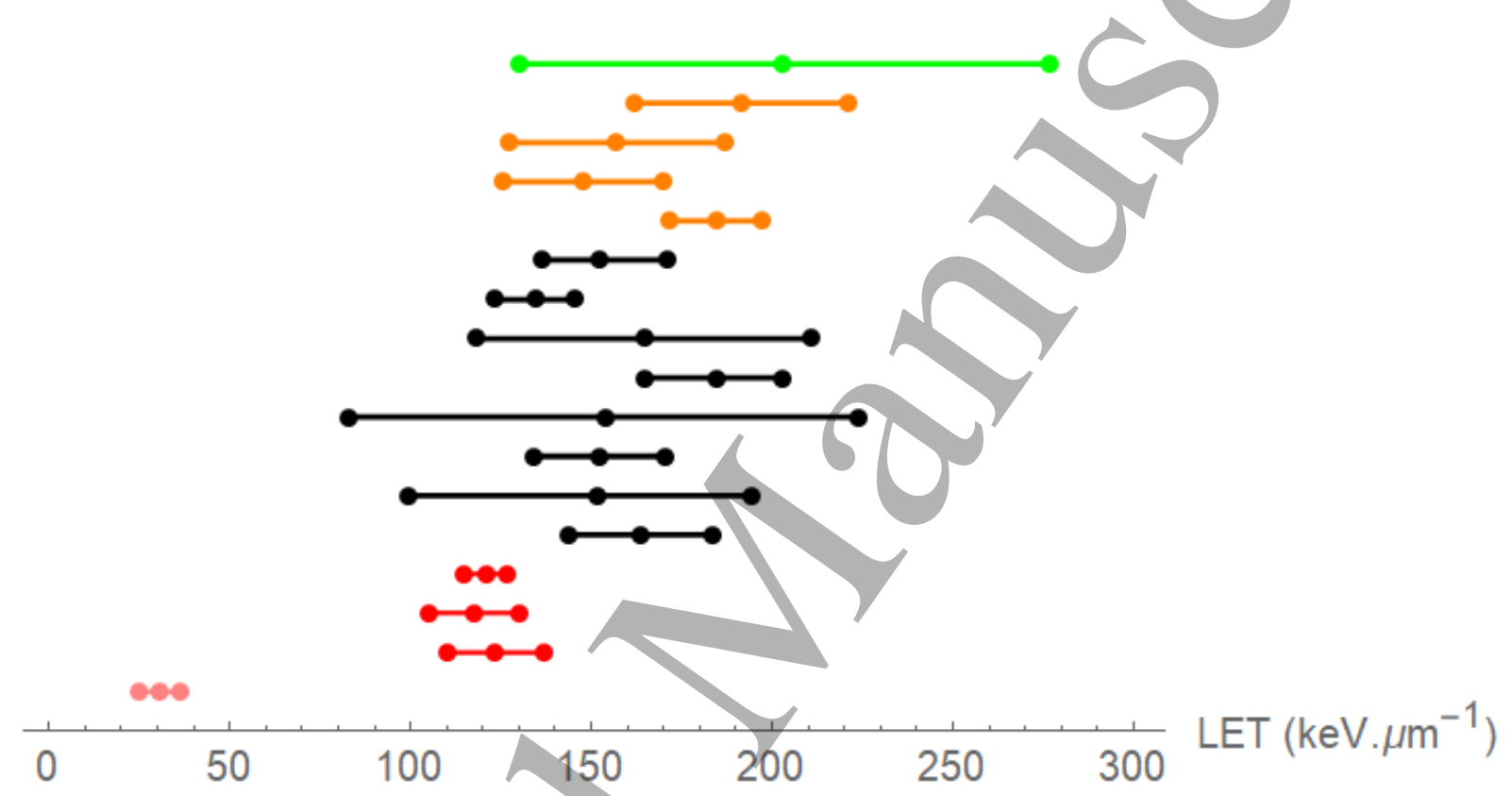

Figure 1. Point estimates for mean LET ${ }_{U}$ values for different ions, shown as central points, with averaged $95 \%$ confidence limits from the two contributing functions shown by the lower and upper limit points. Colours from below to above with data references are for protons (pink [15]), helium [red [2], carbon (black [4, 5]), neon (orange [4]) and argon (green [3]). It should be noted that standard errors would encompass approximately half of the ranges shown above.

The essential kinematic findings are presented in Table 1. The mean LET $_{U}$ value for each ion species is given in results row 1 , which shows the progressive increase in this parameter with $\mathrm{Z}$. This finding is unlikely to be due to chance since the permutations for ordering $\mathrm{Z}$ from lowest to highest lead to a probability of $\mathrm{p}=1 / 5$ ! $(=0.0083)$, although the $95 \%$ confidence limits of the estimates are frequently wide and sometimes overlapping. Table 1 also includes the particle kinetic energies for the $\mathrm{LET}_{\mathrm{U}}$ turnover point values, which also can be displayed graphically as kinetic energy per nucleon (see figure 2). From the unmodified kinetic energies 
values, the particle velocity can be determined (see appendix), as a fraction of the speed of light $(\beta)$ or as a velocity $(v)$. From $\beta$, the effective charge to original charge ratio $\left(Z^{*} / Z\right)$ at LET $_{U}$ was found using the Barkas equation (see appendix). The results show that the charge ratio remains high at 0.99 or larger at $\mathrm{LET}_{\mathrm{U}}$, but these values mark the beginning of their further more rapid reduction at lower $\beta$ values beyond $\mathrm{LET}_{\mathrm{U}}$. Figure 3 shows the overall relationship between $\beta$ and $Z * / Z$. Thus, all ions in the present data set decelerate down to a value of $\beta$ where $Z * / Z$ is around 0.99 at $L_{E T}$, before more rapid change in $Z * / Z$ will occur. This suggests that velocity must reduce to an extent that allows the maximum influence of the highest possible charge on the neighbouring bio-molecules, just before the ion regains electrons and slows further, and so increasing the LET to an extent that reduces RBE by causing 'overkill' due to excess energy deposition beyond what is necessary to cause lethality. These velocities (and particle energies) are typical of those occurring within the Bragg peak regions of the various ions. Figure 4 shows the same relationships with intermediate ion species (each with $\mathrm{Z}$ increments of unity) included.

Table 1. Point estimates of kinematic parameters at the estimated mean LET $\mathrm{U}$ positions.

\begin{tabular}{|c|c|c|c|c|c|}
\hline & $\begin{array}{l}\text { Proton } \\
Z=1, A=1\end{array}$ & $\begin{array}{l}\text { Helium } \\
\mathrm{Z}=\mathbf{2 , \mathrm { A } = 4}\end{array}$ & $\begin{array}{l}\text { Carbon } \\
\mathrm{Z}=6, \mathrm{~A}=12\end{array}$ & $\begin{array}{l}\text { Neon } \\
Z=10, A=20\end{array}$ & $\begin{array}{l}\text { Argon } \\
Z=18, A=40\end{array}$ \\
\hline LET $_{U}\left(k^{\prime}\right.$ V. $\left.\beta m^{-1}\right)$ & 30.43 & 120.66 & 157.10 & 187.8 & 207.9 \\
\hline $\begin{array}{l}\text { Kinetic Energy } \\
(\mathrm{MeV})\end{array}$ & 0.74 & 3.25 & 135 & 685 & 5350 \\
\hline$\beta(=v / c)$ & $0.040[0.015]$ & 0.042 & 0.154 & 0.265 & 0.486 \\
\hline$v\left(\right.$ nm.fs $\left.^{-1}\right)$ & 11.69 & 12.59 & 46.17 & 79.45 & 145.70 \\
\hline $\mathbf{Z} * / \mathbf{Z}$ & $\begin{array}{c}\text { Not } \\
\text { applicable }\end{array}$ & 0.96 & 0.99 & 0.99 & 0.99 \\
\hline$\beta / \mathbf{Z}$ & $0.040[0.015]$ & 0.021 & 0.027 & 0.027 & 0.027 \\
\hline $\begin{array}{l}\text { v/Z (nm.fs }{ }^{-1} \text { per unit } \\
\text { Z) }\end{array}$ & $11.96[4.50]$ & 6.30 & 7.70 & 7.95 & 8.09 \\
\hline$\beta / A$ & $0.040[0.015]$ & 0.011 & 0.013 & 0.013 & 0.012 \\
\hline v/A(nm.fs'-1/nucleon) & $11.96[4.50]$ & 3.15 & 3.85 & 3.97 & 3.64 \\
\hline Index $1=\beta A / Z^{2}$ & $0.040[0.015]$ & 0.042 & 0.051 & 0.053 & 0.060 \\
\hline $\begin{array}{l}\text { Index2=vA/Z } Z^{2}\left(\mathrm{~nm}_{.} \mathrm{fs}^{-}\right. \\
\text {1) }\end{array}$ & 11.69 & 12.39 & 15.39 & 15.89 & 17.99 \\
\hline Katz Ratio $\mathrm{Z} * 2 / \beta^{2}$ & $628[4444]$ & 2111 & 1507 & 1421 & 1355 \\
\hline
\end{tabular}

Comments: The proton data shown within square brackets are for the extrapolated results given in Figure 8. Z, A and $\beta$ are dimensionless quantities. Statistics for (a) Index 1: mean=0.049, median=0.051, $\sigma=0.009, \mathrm{cv}=0.17$ (b) Index 2: mean=14.71, median=15.39, $\sigma=2.56, \mathrm{cv}=0.17$; Note that speed of light is approximately $300 \mathrm{~nm} . \mathrm{fs}^{-1}$. In comparison, the Katz ratio results from figure 5 have summary statistics of: mean=1410, median 1421, $\sigma=518, \mathrm{cv}=0.37$. 
Table 2. Some estimated velocities at LETs of $50 \%$ of LET LET $_{U}$ and $200 \%$ of LET presented in list format for helium, carbon, neon and argon ions respectively.

\begin{tabular}{|c|c|c|}
\hline & $50 \%$ LET $_{U}$ & $200 \%$ LET $_{\mathrm{U}}$ \\
\hline$\beta(=v / c)$ & $\{0.068,0.231,0.374,0.709\}$ & $\{0.017,0.098,0.167,0.319\}$ \\
\hline $\begin{array}{l}\mathrm{v} / \mathrm{Z} \\
\left(\mathrm{nm} . \mathrm{fs}^{-1} \text { per unit }\right. \\
\mathrm{Z})\end{array}$ & $\{10.19,11.52,11.21,11.81\}$ & $\{2.55,4.89,5.0,5.31\}$ \\
\hline $\begin{array}{l}\text { v/A } \\
\text { (nm.fs'-1/nucleon) }\end{array}$ & $\{5.10,5.77,5.61,5.31\}$ & $\{1.27,2.45,, 2.50,2.39)$ \\
\hline $\begin{array}{l}\operatorname{Index} 2=v A / Z^{2} \\
\left(n m . f^{-1}\right)\end{array}$ & $\{20.39,23.08,22.42,26.24\}$ & $\{5.1,9.79,10.01$ \\
\hline
\end{tabular}

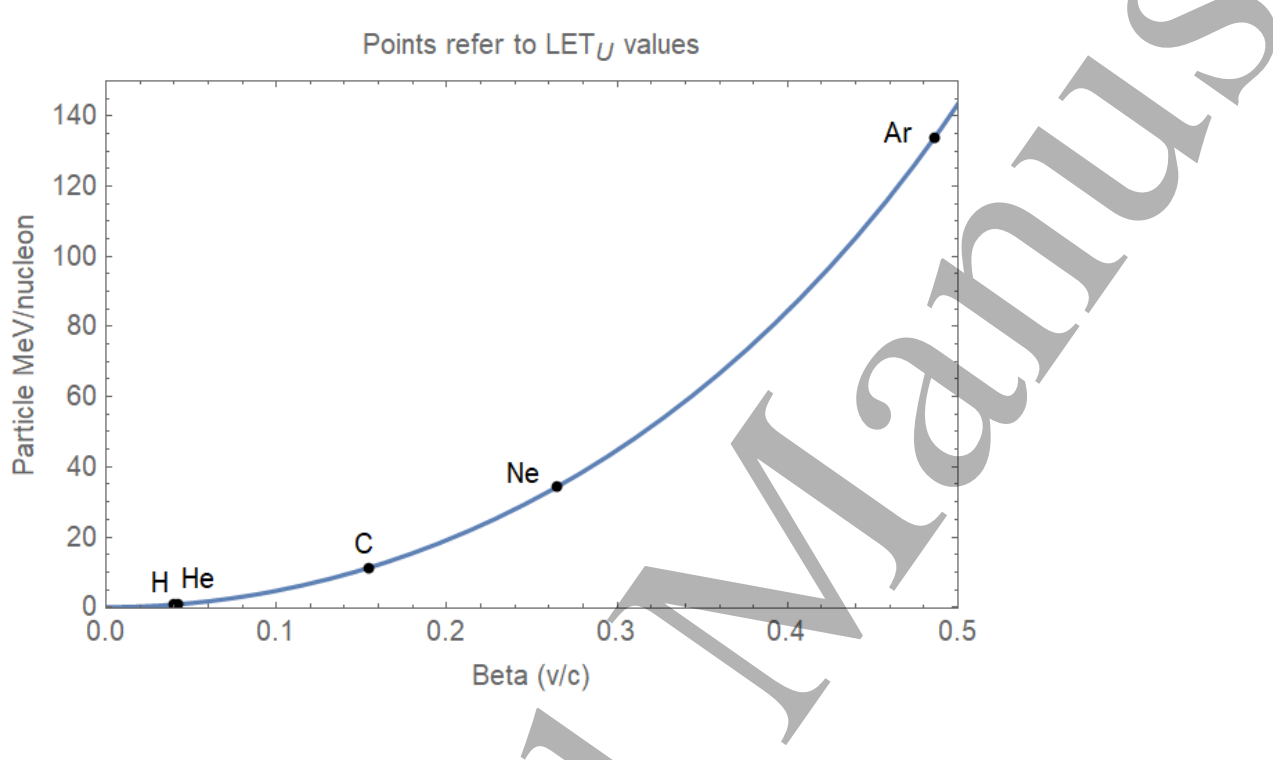

Figure 2. Plot of Particle kinetic energies per nucleon with the relativistic velocity $(\beta)$. The points indicate the values at the estimated LETU turnover positions. 


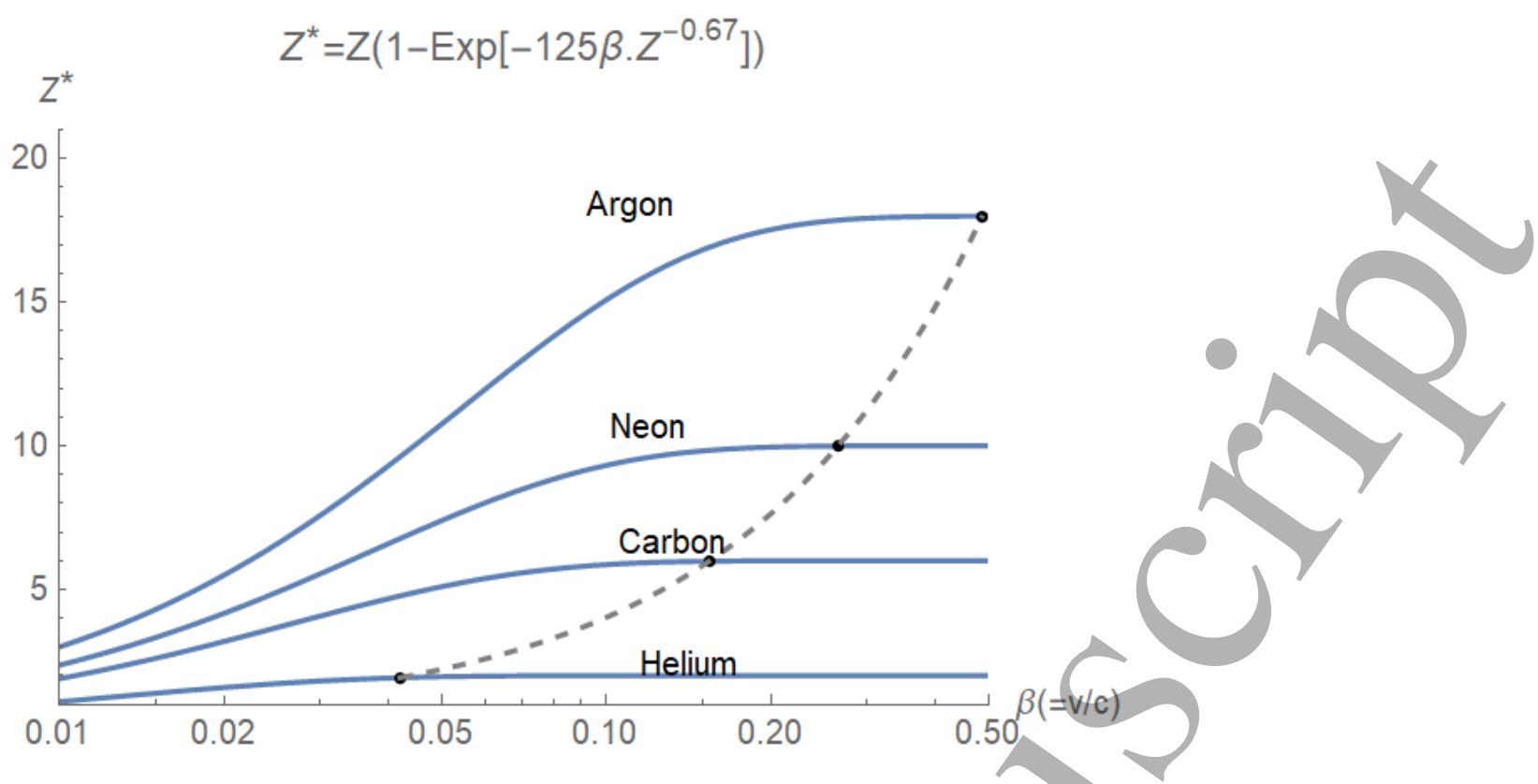

Figure 3: Plot of the relationship between effective value of $Z$, or $\left(Z^{*}\right)$, and the relativistic velocity $(\beta)$ for four ions. The black points are the estimated $Z^{*}$ values at the LET $_{U}$ turnover points. Protons cannot be included here because its own $Z$ value cannot be reduced to a value $<1$. The fitted equation of the hatched curve was obtained by a non-linear equation least squares fit yielding $Z^{*}=0.41+36.16 \beta$, at each LET-RBE turnover point.

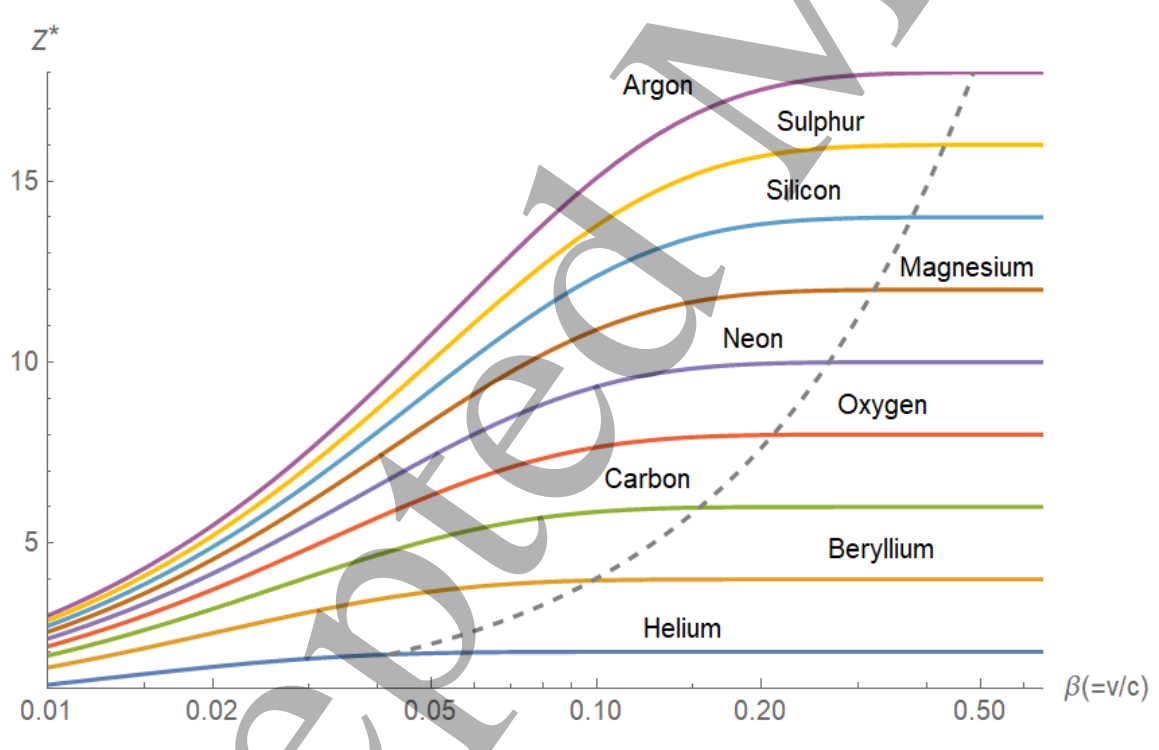

Figure 4. Further examples of the relationship between $\beta$ and $Z * / Z$, for multiple further ions where the hatched curve is the same function used in figure 3 and the crossing point for each curve provides the $\beta$ parameter at the LETU turnover points for each ion, where the $Z * / Z$ value is around 0.99 . 
Substantial changes in the Katz ratio, $Z^{* 2} / \beta^{2}$ at $\operatorname{LET}_{U}$ are shown in figure 5 . The corresponding LET $_{U}$ values do not appear to fall into any simple relationship.

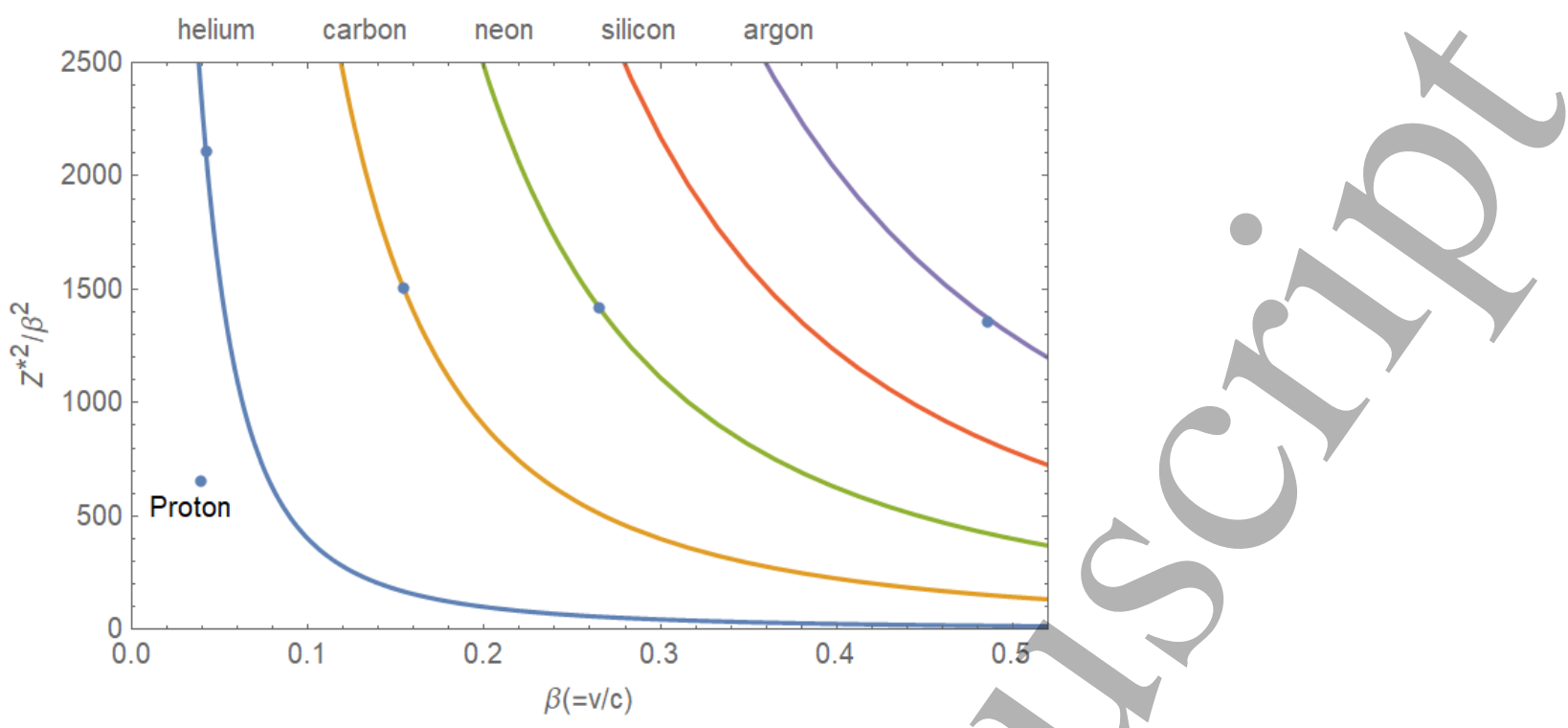

Figure 5. Plot of the Katz $Z^{* 2} / \beta^{2}$ ratio with respect to $\beta$ for five ions, with points showing the values of these parameters at LET $U$ for Helium, Carbon, Neon and Argon. The points refer to the Katz ratio at each LET $_{U}$. The proton case is also shown when $Z$ remains equal to 1 .

In figure $6(\mathrm{a}$ and $\mathrm{b}$ ) the SRIM codes for helium and proton ions are used to generate graphics which show that there is no special mathematical property in the relationship between LET and velocity $(\beta)$ at the $\mathrm{LET}_{\mathrm{U}}$ values. For example, there is no turnover point or inflection point. This finding leads to the possibility that the actual velocity itself may be related to bioeffectiveness. The ions would need to decelerate sufficiently to exert their ionisation effect, mainly on cellular water molecules. The most relevant time frame for completion the physical absorption of radiation energy in biomaterials is the femtosecond [8b]. Also, since cell survival is dependent on multiple DNA strand breakage events accumulating to lethal mutations or chromosomal aberrations, the most relevant distance of action would be the nanometre scale and the dimensions of the DNA helix (see discussion below). Consequently, the $\beta$ velocities were estimated in nm.fs $^{-1}$. The results for each $\mathrm{Z}$ number is given in table 1 (row 3), which shows a wide range. When modified by dividing by Z (row 7) or by A (row9) respectively to obtain the velocities per nuclear proton or nucleon, more uniform results are obtained. Furthermore, several combinatorial indices were sought in an attempt to find a common value at $\mathrm{LET}_{\mathrm{U}}$ for each different ion. The best obtained was for the expression: $\frac{\beta A}{Z^{2}}$ which is dimensionless, or the further expression: $\frac{c \beta A}{Z^{2}}$, which can be regarded in two ways: it 
can either denote the distance traversed in unit time with the other parameters being dimensionless or it can express the distance traversed in unit time with charge ( as expressed by $\mathrm{Z}$ ), but modified by the ratio $\mathrm{A} / \mathrm{Z}$ of the atomic number (or nucleon number) divided by the number of protons within the nucleus, which essentially normalises all values with respect to those of proton and helium ions. The proton and helium values express the index in an unmodified form since $\mathrm{A} / \mathrm{Z}^{2}$ is 1 for both of these ions.

Thus Index 2 yields values in nm per fs with respect to unit charge exposure. The $\mathrm{Z}$ and $\mathrm{A}$ corrected velocities and index 2 have, in the specific context of a biological experiments, relatively stable values.

The coefficient of variation of 0.17 (or $17 \%$ ) for index 1 and 2 is typical of many biological experiments where the degree of heterogeneity is high (different cell types, different laboratories, assay techniques etc), but is substantially less than 0.37 (or $37 \%$ ) for the Katz ratio. It must be remembered that the experiments were designed to demonstrate overall phenomena, and not to provide rigorous statistical estimations of $\mathrm{LET}_{\mathrm{U}}$. Thus further more comprehensive experiments powered to provide much better estimates of LETU, and of the operative gradient parameters are required in order to improve our future understanding of LET-RBE effects.

Figure 6 a
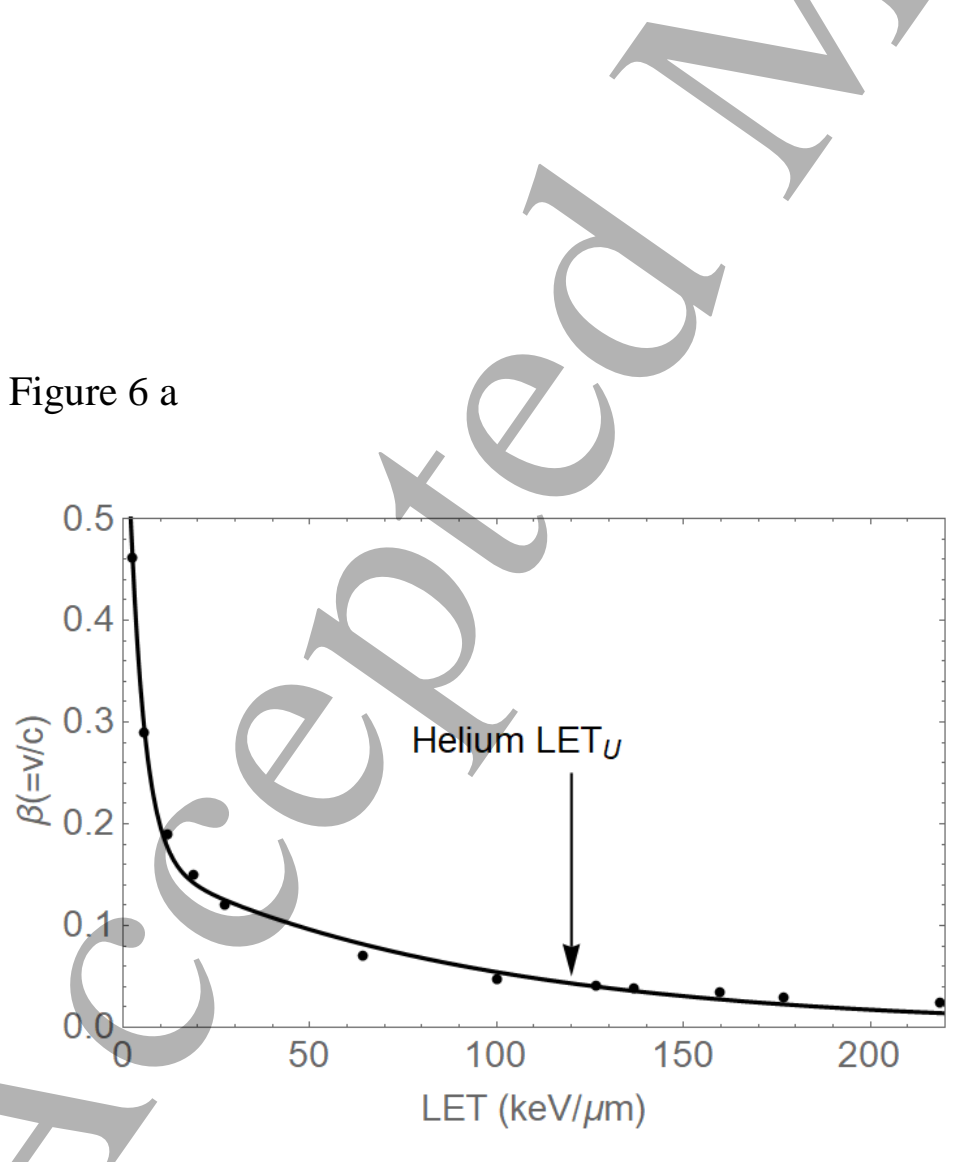
Figure $6 b$

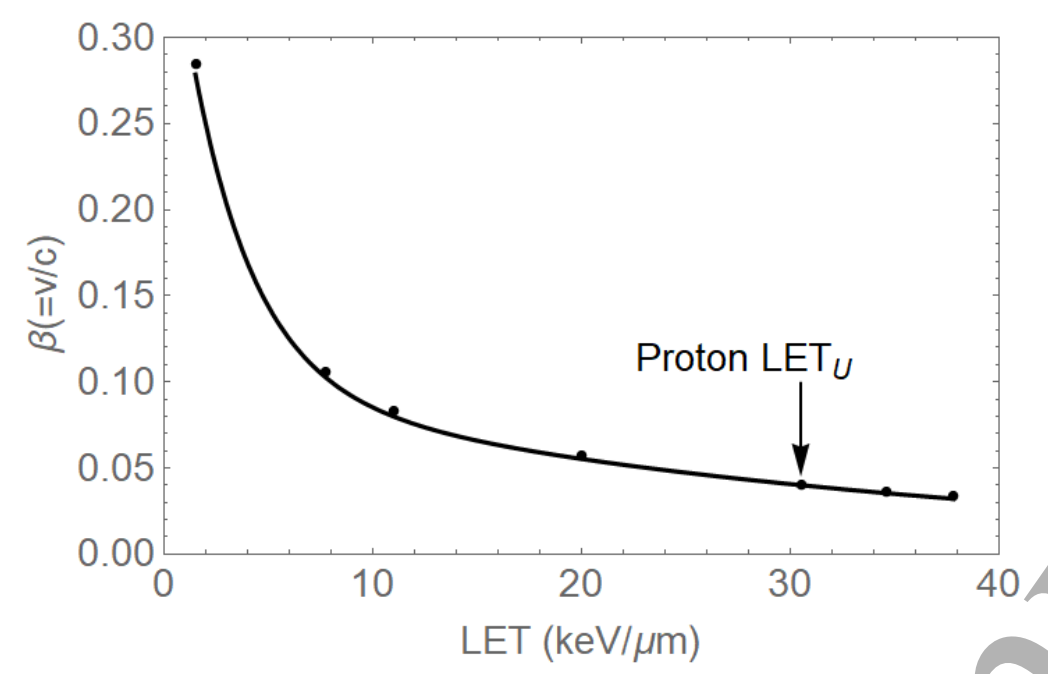

Figure 6 (panels a and b). Plots of $\beta$ with LET for (a) Barandsen`s helium data [2] for a cell survival fraction of 0.8 , showing also the position of the LET-RBE turnover point, and (b) the proton data of Belli et al [ 16 ]. These relationships can be fitted by biphasic equations: $\beta($ helium $)=0.56 e^{-0.251 . L E T}+0.17 e^{-0.0114 . L E T}$,

$$
\beta(\text { proton })=0.3 e^{-0.33 . L E T}+0.1 e^{-0.03 . L E T} \text {. }
$$

The data of Todd [18], can create an inadvertentimpression of an overall optimum bioeffectiveness at LET values of between 100 and $250 \mathrm{keV} . \mu \mathrm{m}^{-1}$ for nine different ions (ranging from Deuterium/Helium to Argon). However the kinetic energies of each ion were around $6.58 \mathrm{MeV}$ per nucleon, which leads to $\beta=0.119,0.119,00.118$ and 0.110 for helium, carbon, neon and argon ions respectively, at LET values of around 165, 220 and $300 \mathrm{keV. \mu m^{- }}$ ${ }^{1}$. These $\beta$ values are all lower than the $\beta$ estimated at each LET $_{U}$ value (shown in Table 1), with the exception of helium which has a higher $\beta$ than at $\operatorname{LET}_{U}$. It is not surprising that the increased RBE noted for the middle of the range ions in his series are for ions where $\beta$ is closest to their LET $_{U}$ values. These are boron, carbon, and nitrogen ions which have estimated $\mathrm{LET}_{\mathrm{U}}$ values of around 163.2, 170.6 and $176.5 \mathrm{keV} . \mu \mathrm{m}^{-1}$ respectively when using the formulation given in the following paragraph. For the other ions (deuterons, helium, lithium, oxygen, neon and argon), the experimental LET values are respectively 6.5, 25, 55, 385 and $1940 \mathrm{keV} . \mu \mathrm{m}^{-1}$, which are either well below their predicted LET $_{\mathrm{U}}$ values (in the case 
of deuterons, helium and lithium), or well above them, resulting in low to intermediate values of RBE.

Figure 7 shows the empirical fit for the $\mathrm{Z}$ and mean estimated $\mathrm{LET}_{\mathrm{U}}$ values, when standardised to the proton value of $30.4 \mathrm{keV} . \mu \mathrm{m}^{-1}$, and can be compared with the previous publications $[11,12]$. The least squares fitted equation is

$L E T_{U}=30.4+\frac{108.4}{0.61}\left(1-e^{-0.61 \sqrt{Z-1}}\right)[\mathrm{Eq} \mathrm{1}]$, which is takes a value of $30.4 \mathrm{keV} . \mu \mathrm{m}^{-1}$ when $\mathrm{Z}=1$ for protons and increasingly larger $\mathrm{LET}_{\mathrm{U}}$ values for increasing $\mathrm{Z}$ numbers.

The use of the square root of Z (or Z-1) in this equation which is standardised to the proton, allows a better upward tapering of $\mathrm{LET}_{\mathrm{U}}$ with $\mathrm{Z}$ towards an upper limit. The previous model (without the square root function) inevitably had a rather flat upper limit which depended on the highest $\mathrm{Z}$ value in the data set used. The justification for the square root function is given in the appendix. Should the proton LET $_{U}$ value be higher (see below) the form of the equation will not change much due to the steepness of the function at $\mathrm{Z}=1$.

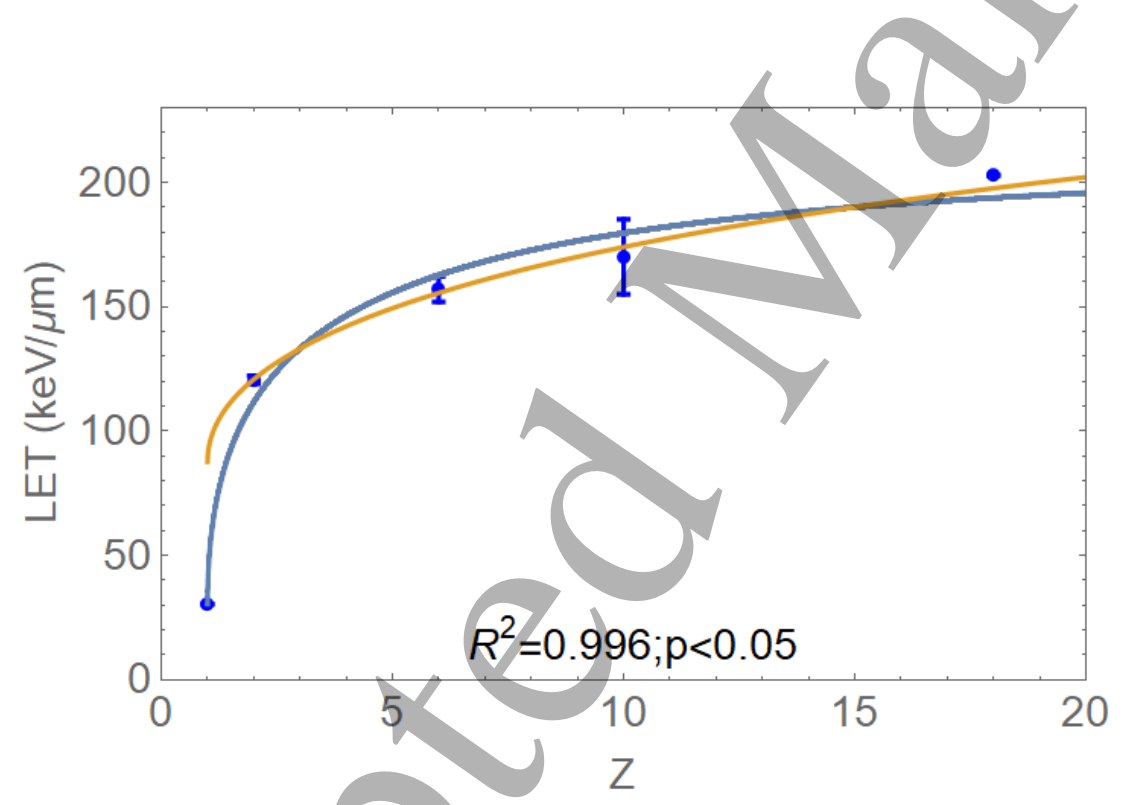

Figure 7: Empirical non-linear fit between $Z$ and $L E T_{U}$, normalised to protons, using $30.4+108.4 / 0.61 \operatorname{Exp}\left[1-0.61(Z-1)^{0.5}\right]$, based on the data of Belli et al [15] and displayed as the darker curve. The lighter curve is based on a proton LET $\mathrm{U}$ of 87.7 $\mathrm{keV} \cdot \mu_{\mathrm{m}}^{-1}$ derived below in the text. Points refer to mean estimated LETU values with standard errors of the mean for $Z=2,6$ and 10 only. It should be noted that the $95 \%$ confidence limits given in figure 1 are much larger than these SEM values.

It is interesting to consider two data sets (from the PIDE data set), in which a turnover point is not so easily identified due to lack of data points. The Silicon and Iron data of Tsuruoka et 
al [19], as shown in figures 8 (a \& b), can be interpreted using the above models. The black point is the predicted LET $_{U}$ value (using equation 1). The initial gradient is based on the approximate starting point of $\mathrm{RBE}=1$ when $\mathrm{LET}=1$ and the first six grey data points and fitted with an equation 1+m.LET (see appendix) ; the predicted LET $_{U}$ (black point) and the seventh (highest) LET data point is fitted by $1+\mathrm{c} / \mathrm{LET}$. For Iron ions, the data is distributed only above the predicted LET $_{U}$ vale using equation 1 . The first data point $(\mathrm{LET}=200)$ is excluded and the remaining data fitted by $1+\mathrm{c} / \mathrm{LET}$, which provides an estimated intersection point with a linear function which fits the $\mathrm{LET}+1$ and $\mathrm{RBE}=1$ condition and the first data point at LET $=200$. The intersections of the data-fitted lines and curves provide LET $_{U}$ values of 185.89 and $215.56 \mathrm{keV} . \mu \mathrm{m}^{-1}$ for Silicon and Iron ions respectively, compared with the predicted values of 189.19 and $199.95 \mathrm{keV} . \mu \mathrm{m}^{-1}$ obtained using equation 1.

Figure 8(a)

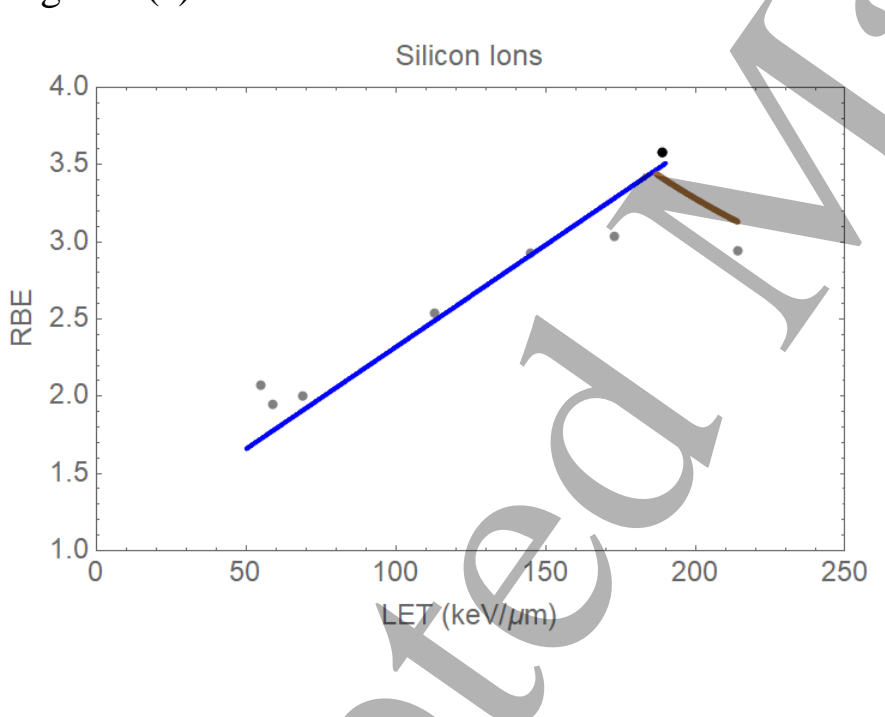

Figure $8(b)$ 


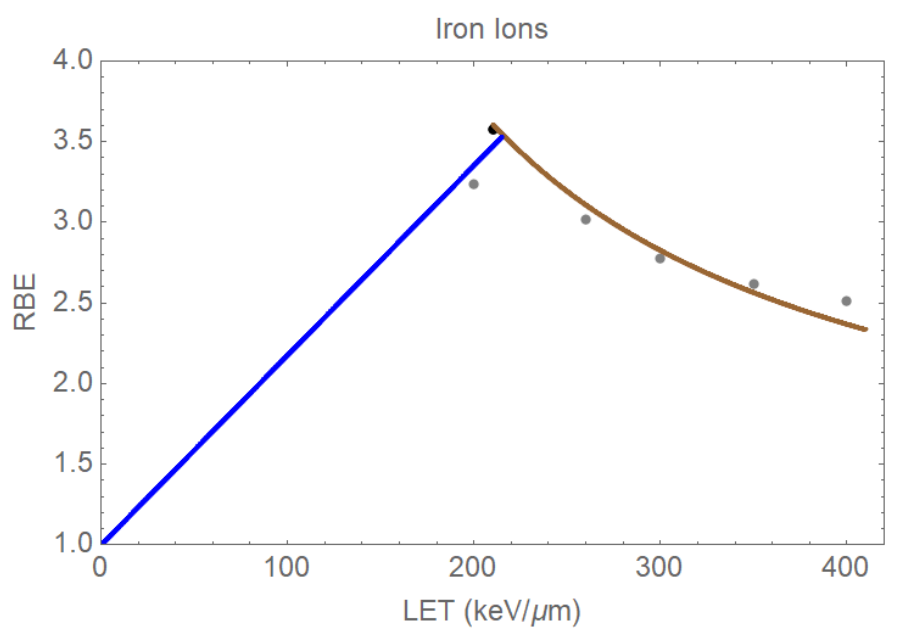

Figure 8 (a\&b). Plots of data from reference 19 for (a) the Silicon data, (b) the Iron data. In each case the intersection point of the two fitted functions provides an estimate of LET $U$ which is close to the dark point obtained from the predictive equation [1].

The data contained in table 1 shows that the proton seems to have anomalous kinematics at LET $_{U}$. The estimated proton velocities are very close to those of helium ions. This might be for physical reasons, but it is important to consider if the input data for $\mathrm{LET}_{\mathrm{U}}$ [15] is correct.

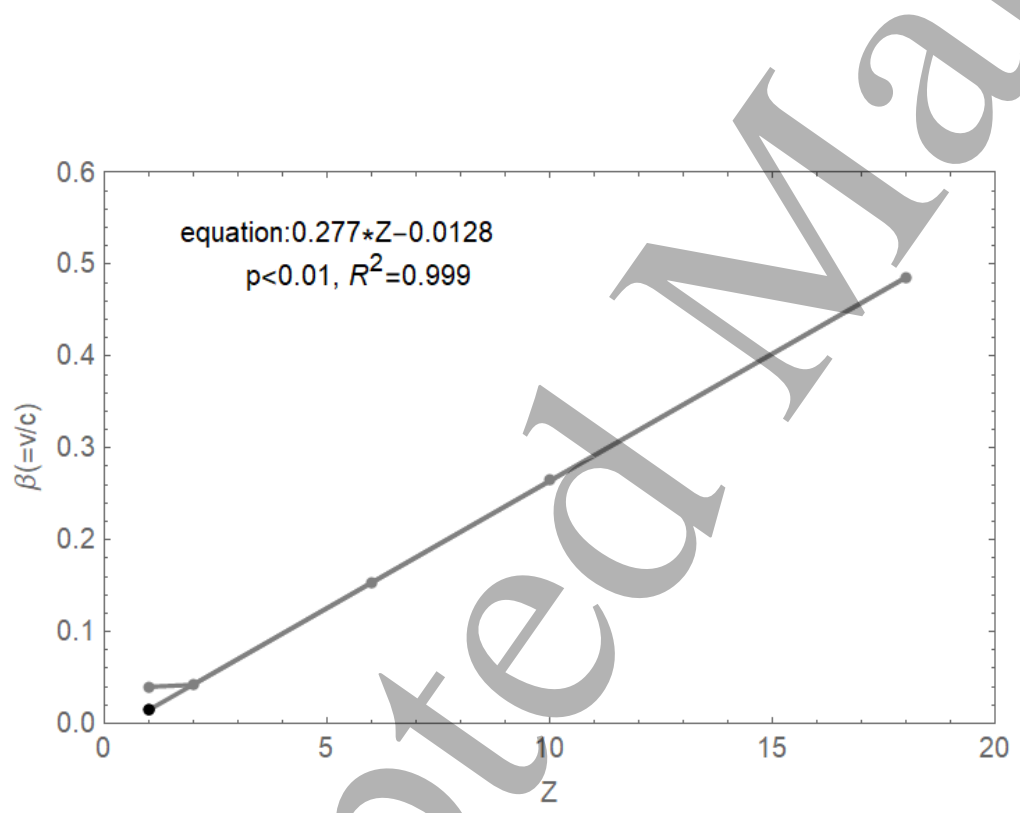

Figure 9. Estimated $\beta$ velocity parameter values at the LETu turnover points given in table 1 are plotted as grey points against $Z$ number for protons, helium, carbon, neon and argon ions. The black point is obtained by extrapolating the line fitting the ions through helium to argon only, and provides a theoretical $\beta$ value for $Z=1$ (arguably for deuterium) at LETU, since the line contains only ions that contain neutrons. The proton and helium velocities are very similar but requires a step function to the linear relationship for higher $Z$ value ions. 
Figure 9 shows the deviation of the proton $\beta$ value from the linear function, which fits the other heavier ions by extrapolation of the line down to the $\mathrm{Z}=1$ value, where $\beta$ is smaller than the experimental proton value. The linear fit provides an alternative $(Z=1) \beta$ value of around 0.015 , which indicates a kinetic energy of either around $0.105 \mathrm{MeV}$ (proton) or $0.21 \mathrm{MeV}$ (deuterium) and, using the SRIM software, the LET $_{\mathrm{U}}$ is then estimated as around 87.7 $\mathrm{keV} \cdot \mu \mathrm{m}^{-1}$ (proton) or $86.5 \mathrm{keV} \cdot \mu \mathrm{m}^{-1}$ (deuterium), which is substantially higher than the value of $30.4 \mathrm{keV} . \mu \mathrm{m}^{-1}$ estimated by Belli et al [15]. However, if this value is only correct for deuterium this might better explain the discrepancy of other estimates that the proton RBE is between 55 and $80 \mathrm{keV} . \mu \mathrm{m}^{-1}$ (as shown below in discussion section using references 21,23 , 24), because these experiments contained an increasing proportion of deuterium ions for higher LET conditions, for good reasons connected with particle range in the experimental set-ups [B Vojnovic, personal communication 2019]. So, it seems reasonable to suggest that the $30.4 \mathrm{keV} . \mu \mathrm{m}^{-1}$ proton value remains correct, because increasing use of deuterons instead of protons (in mixed proton and deuteron experiments) would increase the estimated LET $_{U}$ value towards the range quoted above. This is further supported by the values of the index $\beta \mathrm{A} / \mathrm{Z}^{2}$ being almost identical for protons at $30.4 \mathrm{keV} \cdot \mu \mathrm{m}^{-1}$ and deuterons around $70 \mathrm{keV} \cdot \mu \mathrm{m}^{-1}$ (see table 3), which is in the range (0.04-0.06) given in table 1 for LET $_{U}$. The further implication is that the straight line fit shown in figure 9 applies only to charged particles which contain neutrons, possibly due to further nuclear effects, which do not apply to the same extent for protons.

Table 3: Kinematic comparisons of protons and deuterons.

\begin{tabular}{|l|l|l|l|l|l|l|}
\hline & \multicolumn{3}{|c|}{ Protons } & \multicolumn{3}{c|}{ Deuterons } \\
\hline $\begin{array}{l}\text { LET } \\
\left(\mathrm{keV} . \mu \mathrm{m}^{-1}\right)\end{array}$ & $\begin{array}{l}\text { Relativistic } \\
\text { velocity }(\beta)\end{array}$ & $\begin{array}{l}\text { Energy } \\
(\mathrm{meV})\end{array}$ & $\begin{array}{l}\text { Index } \\
\beta \mathrm{A} / \mathrm{Z}^{2}\end{array}$ & $\begin{array}{l}\text { Relativistic } \\
\text { velocity }(\beta)\end{array}$ & $\begin{array}{l}\text { Energy } \\
(\mathrm{meV})\end{array}$ & $\begin{array}{l}\text { Index } \\
\beta \mathrm{A} / \mathrm{Z}^{2}\end{array}$ \\
\hline 30.4 & 0.04 & 0.74 & $\underline{0.04}$ & 0.04 & 1.45 & 0.08 \\
\hline 60 & 0.023 & 0.25 & 0.023 & 0.023 & 0.5 & 0.046 \\
\hline 70 & 0.02 & 0.19 & 0.02 & 0.02 & 0.38 & $\underline{0.04}$ \\
\hline 80 & 0.017 & 0.14 & 0.017 & 0.017 & 0.28 & 0.034 \\
\hline
\end{tabular}

Predictive models which use the scaling of LET with RBE, or the separate gradients of the $\alpha$ linear quadratic model radio-sensitivity parameter, as originally suggested by Wilkens and Oelfke [20] must be based on reliable LET information. There are important implications for 
models that need an accurate estimation of proton LET $_{U}$ values [11-14]. For example, in the case of the proton LET $_{\mathrm{U}}$ being either 30.4 or as high as $87.7 \mathrm{keV} . \mu \mathrm{m}^{-1}$ (the probable deuterium value), the estimated RBE increment above unity would be reduced by the ratio 30.4/87.7 (= 0.347), or more approximately by a third. In this way, an RBE estimated of say 1.3 would be reduced to $1+0.3 \times 0.33$, to approximately 1.1 . Further detailed experiments are required to confirm the proton $\mathrm{LET}_{U}$ value.

\section{Discussion}

The phenomenon of a turnover point of RBE with LET is well established, although it was demonstrated in many experiments in different laboratories, with different cell lines/tissues and accelerator systems. For a considerable time there was no universal/agreement about where maximum bio-efficiency occurred with respect to LET. For example, Berry [21] concluded that the Hammersmith experiments (using fast neutrons which predominantly ionise by means of recoil protons and must be relevant for the proton $\mathrm{LET}_{U}$ ) had the greatest RBE values at between $60-80 \mathrm{keV} \cdot \mu \mathrm{m}^{-1}$, whereas for a range of heavier ions a maximum RBE occurred at 110-220 keV. $\mu \mathrm{m}^{-1}$. Some textbooks have emphasised generic conclusions, largely emerging from the impressive experiments of/Barendsen [8a, 22] with deuterons and $\alpha$-particles, where the $\mathrm{LET}_{\mathrm{U}}$ appeared to be around $110-120 \mathrm{keV} . \mu \mathrm{m}^{-1}$. The spacing of ionisation happens to match the DNA helix dimensions for $\alpha$-particles at around $110 \mathrm{keV}$. $\mu \mathrm{m}^{-1}$ [8a], but does this apply to all ions? The data of Todd [18] can inadvertently mislead by suggesting that the highest RBE is obtained only for LET values between 100 and $200 \mathrm{keV}$. $\mu \mathrm{m}^{-1}$. Such an impression is probably due to the mono-energetic nature of the experiment, so that many of the ions have experimental LET values which are either well below or above their LET $_{U}$ values, so that their RBE`s are low when compared to those ions that have experimental LET values closer to their predicted LET $_{U}$ values at the energy of the experiment. Reasonable predictions of the Todd`s results can be found when using the LET $_{U}$ based simple efficiency model where each ion has its own unique LET value [11].

There has been a longstanding controversy regarding the LET $_{U}$ of protons in the literature. The data discussed by Berry [21], already mentioned above, suggests that the proton LET $_{U}$ might be between $60-80 \mathrm{keV} . \mu \mathrm{m}^{-1}$. Further experimental data presented by Perris et al [23], who also collated the work of other authors, and also the separate studies of Folkard et al [24] 
suggest that the proton $\mathrm{LET}_{\mathrm{U}}$ could be around $55-65 \mathrm{keV} . \mu \mathrm{m}^{-1}$, although higher values have not been excluded. It has been mentioned already that the use of deuterons may have influenced these conclusions. Many experimental proton publications do not include sufficient variation in LET values to determine $\operatorname{LET}_{\mathrm{U}}$, as for example the data of Britten et al 2013 [25].

However, the linearity of the LET ionisation measurement has been criticised, with preference given to lineal energy which accounts for ionisation over a spherical volume around each track, which is considered to be more biologically relevant [1b]. Katz and others suggested that, instead of LET, functions containing the expression $Z^{* 2} / \beta^{2}$ should be used, where $\beta$ is the relativistic velocity (v/c), which correlates with the radius of the cylinders of delta rays which form around particle tracks. Waligorski has reyiewed and extended the Katz ideas by modelling RBE changes with LET [26], although the predicted symmetry of these relationship do not appear to agree with the hypothesis that the $\mathrm{LET}_{U}$ positions are unique for each ion species.

The speculative kinetic information presented in the present study, where the v/Z and v/A ratios and the specified index 1 yields a reasonably stable value at LET $_{U}$ for the different ions, is worthy of further research. Since the LET $U$ values have been derived from biological data sets, considerable variation due to biological heterogeneity is to be expected. The critical distance of around 6-8 nm fs ${ }^{-1}$ per unit Z or 3-4 $\mathrm{nm} \mathrm{fs}^{-1}$ per nucleon (a duration of time that would allow the initial physico-chemical reactions required for ionising radiation to exert bioeffects) is of considerable interest. In terms of DNA structure this distance exceeds the double stranded DNA helix (2nm) but conforms better with the size and shape of the nucleosome. The latter has dimensions of a $6 \times 6 \times 11 \mathrm{~nm}$ rectangular solid, the short diagonal will be around $8.49 \mathrm{~nm}$ and the longer internal diagonal around $13.89 \mathrm{~nm}$, so that all the $\mathrm{nm}$ distances given in table 1 seem to be very relevant, providing distances of action that will either partially or completely include a nucleosome, depending on the beam trajectory. Such findings may be coincidental, but must be considered relevant to the dimensions of DNA, especially for long patch damage or eventual ultrastructural chromosomal damage to cause cellular lethality. They are however, speculative.

Alper reviewed the difficulties associated with the calculation of radiation targets or associated macromolecular size [1c]. Roots and Okada [27] found a $6 \mathrm{~nm}$ target size for the production of 
X-ray induced DNA double strand breaks, which would encompass the range of the radiolysis of water hydroxyl radical, but a larger distance of $9 \mathrm{~nm}$ for experimental cell survival. Further progress was made using Monte Carlo simulations by Goodhead and Brenner [28], who found that the rare occurrence of greater than $100 \mathrm{eV}$ of low LET radiation to a $3 \mathrm{~nm}$ diameter sphere correlated best with cell killing efficiency. For higher LET radiations, Goodhead and Charlton [29] estimated that energies greater than $340 \mathrm{eV}$ was required within a $5 \mathrm{~nm} \times 10 \mathrm{~nm}$ length cylindrical surface when using alpha-particles. These considerations apply to the spatial formation of DNA double strand breaks, although to cause cell killing many such lesions are required to form lethal chromosomal lesions. The increased efficiency of cell killing, expressed as radiosensitivity, with high LET radiations requires enhanced clustering of events, resulting in multiple DSB and more complex DNA disorder in a critical vicinity [30].

Another aspect which should be considered by theoreticians and experimentalists is the near instantaneous inter-track distance (independently of overall dose), since it seems to influence experimental RBE results when different beam delivery techniques are used: RBE falls with distance in pre-scattered beams, but not with scanned beams where inter-track distances are well maintained [14, 31]. Pohlit [32] showed no such interaction at the physical level of damage production, but suggested that nonlinear dose-related bio-effects could follow repair/misrepair and lesion fixation.

A further approach has recently been proposed by Luhr and colleagues [33], who used the expression $\mathrm{Q}=\mathrm{Z}^{2} / \mathrm{E}$, to define radiation quality, where $\mathrm{E}$ is the kinetic energy in $\mathrm{MeV}$ per nucleon. The $\mathrm{Q}$ value does influence the $\alpha$ parameter and to a much smaller extent the $\beta$ parameter of the linear-quadratic model of radiation effect and so correlates with relative biological effectiveness in their preliminary findings, but the model does not allow for a turnover point of RBE with LET at very low kinetic energies.

The $\mathrm{LET}_{\mathrm{U}}$ parameter has received scant attention in the past, but it can now be appreciated that it is highly relevant to RBE considerations. It must be noted that LET at selective points within a bio-system will be averaged values, due to some variations in particle energies as they emerge from an accelerator and beam delivery system, as well as the process of straggling during their traversion through matter. There will inevitably be errors in the physical parameters as well the bio-parameters, and this topic merits further research. The estimation of LET at different energies has changed in subtle ways over the past fifty years 
[16], which covers the time interval over which the classical LET and RBE experiments were done.

This paper has identified some of the physical characteristics associated with maximum bioefficiency. Beyond these, biology will inevitably take over with provision of multiple further constraints that determine cell killing efficiency and with increasing LET the phenomenon of 'overkill' at the $L E T_{U}$ value. These include the capacity of individual sections of DNA within a nucleosome to repair (using different repair pathways) and so reduce the yield of mutations and/or gross chromosomal rearrangements that confer lethality, as well as the concept of 'wasted dose' where if excess energy is deposited locally there can be no increment in cell kill (as for example the case if the same chromosome has two lethal aberrations rather than one). The relativistic velocity differences between deuterons and protons is intriguing: the higher $\beta$ velocity value for protons suggests that mass is influential even when charge is the same. These differences could be due to a thermal pressure wave effect caused by the greater mass of deuterons, although this is highly speculative.

The present work is limited by the small numbers of data points in most available data sets and there is some concern as to the true LET $\mathrm{U}$ value of protons. Further experiments are urgently indicated, preferably in a dedicated international biophysics laboratory, which could investigate some of the predictions generated in the present article.

In this way, the best overall model for the estimation of RBE with changes of LET and dose in various ion species can be found. The existing LEM (with mechanistic overkill and extrapolated linear cell survival at high dose)[34] and MKM models [35] (with an empirical saturation correction but applied only to the $\alpha$ radiosensitivity) must also be rigorously tested since they involve different assumptions to those in the present paper. Another assumption made in the present paper about the linearity of RBE increasing with LET, although confirmed in many studies, has been criticised elsewhere [36], although experimental conditions for the precise placement of cells relative to Bragg peaks may account for nonlinearity, hence the need for rigorous experiments under standardised conditions with very precise beams rather than in clinical facilities.

The scope for research is considerable in this important field of Science with applications to Medicine.

\section{Summary}


Each ion probably has a unique Z-dependent $\operatorname{LET}_{U}$ value, where there is a unique $\beta$ velocity parameter which corresponds to the ion retaining around $99 \%$ of its charge, and where the velocity per nucleon is around 3-4 nm.fs ${ }^{-1}$.

At LET values below LET $_{U}$, bio-effectiveness increases with LET, as $\beta$ falls progressively until the critical distances and time frame for optimum radiochemical events at the LET $\mathrm{U}$ point is reached. At even higher LET values, bio-effectiveness reduces when the ions begin to gain more electrons but also slow down further so that the further shortened nm distances become contribute to reduced bio-effectiveness. Improved knowledge of LET $U$ for each ion can in principle be used for predictive modelling of $\mathrm{RBE}$, which has applications in particle radiotherapy, radiation protection including space travel.

Advances in Knowledge: This study enhances the limited prior knowledge about the physical conditions of particle beams that provide maximum bio-effectiveness. These data can be used for predictive modelling of RBE.

Acknowledgements: The authors are especíally grateful for discussions regarding RBE and LET with colleagues such as Boris Vojnovic, Peter O Neill and Dudley Goodhead.

Declarations: None

\section{Appendix}

1. The Barkas formula

$$
Z^{*}=Z\left(1-e^{\left[-125 \cdot \beta \cdot Z^{-0.67}\right]}\right) \quad[\mathrm{A} 1]
$$

2. The Katz formula: to account for $\delta$-rays ejected from tracks. These $\delta$-rays are radially distributed and responsible for most bio-effects and ionisations collected by detectors. With considerable acumen, Katz (1970) proposed a function containing $\frac{\left(Z^{*}\right)^{2}}{\beta^{2}}$, where $Z^{*}=$ effective nuclear charge of atomic nucleus of atomic charge $\mathrm{Z}$ and $\beta$ is the relativistic velocity $(v / c)$, in order to estimate the effective radius of ionisation and which should correlate with bio-effectiveness. As fully stripped ions slow down through matter, they pick up electrons while causing ionisation, so $\mathrm{Z}^{*}$ becomes less than Z, the parameter will change with depth.

3. Kinetic energy at relativistic energies $=m c^{2}\left(\frac{1}{\sqrt{\left(1-\beta^{2}\right)}}-1\right)$

4. Data fitting equations: 
The simple energy efficiency model [11-14], with its dependency on $\mathrm{Z}$ to determine LET $_{U}$ includes the following equations:

The operative LET value is $L E T_{x}$, and $L E T_{C}$ is the $\mathrm{LET}$ of the reference radiation for RBE, studies.

The $\alpha$ value at LET $\mathrm{U}$ is $\alpha_{u}$, and at the reference LET value is $\alpha_{L}$.

Empirical fits provide a relationship between $\alpha_{L}$ and $\alpha_{U}$, as

$\alpha u=10.57 / 3.92\left(1-\operatorname{Exp}\left[-3.92 \alpha_{L}\right]\right)$, where the initial ratio represents the maximum possible value of $\alpha_{U}$ in units of $\mathrm{Gy}^{-1}$.

this is sufficient for RBEmax estimations where RBEmax $=\alpha_{H} / \alpha_{L}$, sometimes referred to as $\mathrm{RBE}_{\alpha}$

The $\alpha$ value at the operative LET is then given by

$$
\alpha_{H}=\alpha_{L}+\frac{L E T X-L E T_{C}}{L E T_{U}-L E T C} .\left(\alpha_{U}-\alpha_{L}\right)
$$

for all values of $L E T_{x}<L E T_{U}$. This represents a linear increase in radiosensitivity with LET.

Beyond $L E T_{U}$, the $\alpha$ values, (due to 'wasted energy' at $L E T_{x}$ ) reduce with LET and are estimated by an inefficiency function as:

$$
a_{H}=\alpha_{L}+\left(1-\frac{L E T X-L E T_{U}}{L E T_{x}-L E T_{C}}\right) \cdot\left(\alpha_{U}-\alpha_{L}\right)
$$

For experiments where RBE results are available, a similar approach is used with the reference RBE being 1 and by using simpler symbolism, with a linear relationship for LET values up to $\mathrm{LET}_{\mathrm{U}}$, and where $x$ replaces $\mathrm{LET}_{\mathrm{X}}$-LETC, $U$ replaces $\left(\mathrm{LET}_{\mathrm{U}}-\mathrm{LET}_{\mathrm{C}}\right), \triangle R B E$ is the increment in RBE, equation A3 can be replaced as

$$
R B E=1+\frac{x}{U} \Delta R B E
$$

Or more simply as $R B E=1+m x$,

where $m$ is the gradient,

The LET-RBE relationship beyond LET $_{\mathrm{U}}$ is changed from equation A4 by further replacements. These assume that since $L E T_{x}$ is then large compared to $L E T_{C}$, that $L E T_{x}-L E T_{C}$ can be replaced by $x$ and $L E T_{U}$ by $u$ ( since $L E T_{C}$ is small compared to $L E T_{U}$ ), then

$$
\left.R B E=1+\left(1-\frac{(x-u)}{x}\right) \Delta R B E\right)
$$

$$
\text { Which simplifies to be }
$$

$$
R B E=1+\frac{u}{x} \triangle R B E
$$

And more simply as

$$
R B E=1+\frac{k}{x}
$$

Where the coefficient $\mathrm{k}$ determines the displacement of the reciprocal function.

Equations A6 and A9 can be used to fit LET- RBE data sets, and estimate $m$ and $k$.

The intersection point where $\mathrm{LET}=\mathrm{LET}_{\mathrm{U}}$, is then found by equating A6and A9: 
$1+m L E T_{U}=1+\frac{k}{L E T_{u}}$

Then,

$L E T_{U}=\sqrt{\frac{k}{m}}$

Now $k / m$ will be related to $\mathrm{Z}$ in order for there to be a specific $\mathrm{LET}_{\mathrm{U}}$ for each $\mathrm{Z}$, then

$\operatorname{LET}_{U}=f(\sqrt{Z})$

Where $f(\sqrt{ } Z)$ is some more complex function that contains $\sqrt{ } Z$. This is relevant to the equation that links LET $_{\mathrm{U}}$ to $\mathrm{Z}$ in text (equation 1).

\section{References}

1. Alper T (1979) pp (a) p114-115, (b) p109-110, (c) p26-31 in: Cellular Radiobiology. ISBN 0521294797. Cambridge University Press.

2. Barendsen GW. Responses of cultured cells, tumours and normal tissues to radiations of different linear energy transfer. Curr Topics Radiat Res Q 1968: 4; 293-356.

3. Blakely EA, Tobias CA, Yang TCH, et al. Inactivation of human kidney cells by highenergy monoenergetic heavy-ion beams. Radiat Res. 1979;80:122-60.

4. Furusawa Y, Fukutsu K, Aoki M, Itsukaichi H, Eguchi-Kasai K, Ohara H et al. Inactivation of aerobic and hypoxic cells from three different cell lines by accelerated (3)He-, (12)Cand (20)Ne-ion beams. Radiat Res. 2000 Nov;154(5):485-96. Erratum in: Radiat Res. 2012 Jan;177(1):129-31.

5. Weyrather WK, Ritter S, Scholz M, Kraft G, 1999. RBE for carbon track-segment irradiation in cell lines of differing repair capacity. Int J Radiat Biol. Nov;75(11):1357-64.

6. Joiner MC (2002). Particle beams in radiotherapy page 207. In Basic Clinical Radiobiology Ed GG Steel, Arnold, London.

7. Brahme A. Recent advances in light ion radiation therapy. Int J Radiat Oncol Biol Phys. 2004 Feb 1;58(2):603-16.

8. Hall EJ \& Gaccia AJ (a\&b), (a) page 109 in Radiobiology for the Radiologist, (b) page 10, in $8^{\text {th }}$ Edition (2019) of Radiobiology for the Radiologist. Pub: Walters Kluwer, USA.

9. Friedrich T, Scholz U, Elsässer T, Durante M, Scholz M, (2013). Systematic analysis of $\mathrm{RBE}$ and related quantities using a database of cell survival experiments with ion beam irradiation. J Radiat Res. May;54(3):494-5

10. Sorensen BS, Overgaard/J and Bassler N (2011). In vitro RBE-LET dependence for multiple particle types. Acta Oncol. Aug;50(6):757-62.

11. Jones B (2015) A Simpler Energy Transfer Efficiency Model to Predict Relative Biological Effect (RBE) for Protons and Heavier Ions. Frontiers of Oncology. 2015 Aug 11;5:184. doi: 10.3389/fonc.2015.00184. Erratum in: Front Oncol. 2016;6:32.

12. Jones B. Towards Achieving the Full Clinical Potential of Proton Therapy by Inclusion of LET and RBE Models. Cancers 2015, 7, 460-480. 
13. Jones B Clinical radiobiology of proton therapy: modeling of RBE. Acta Oncol. 2017 Nov;56(11):1374-1378

14. Jones B, McMahon SJ, Prise KM. The Radiobiology of Proton Therapy: Challenges and Opportunities Around Relative Biological Effectiveness. Clin Oncol(R Coll Radiol). 2018 May;30(5):285-292.

15. Belli M, Bettega D, Calzolari P, Cera F, Cherubini R et al 2000, Inactivation of human normal and tumour cells irradiated with low energy protons. Int J Radiat Biol. 2000 Jun;76(6):831-9.

16. SRIM - The stopping and range of ions in matter. Chapters 3 and 4 in: Ziegler JF, Biersack JP, and Ziegler MD . Pubs: SRIM Co. Chester, MD, USA, (2008).

17. H Barkas, Nuclear Research Emulsions, Vol 1, chapter 9, p 371, Academic Press, New York, 1963

18. Todd P, 1967. Heavy-ion irradiation of cultured human cells. Radiation Research, 7, 196207.

19. Tsuruoka C, Suzuki M, Kanai T, and Fujikata K (2005). LET and Ion Species Dependence for Cell Killing in Normal Human Skin Fibroblasts. Radiation Research 163, 494-500.

20. Wilkens JJ, and Oelfke U (2004). A phenomenological model for the relative biological effectiveness in therapeutic proton beams. Phys Med Biol (2004) 49(13):2811-25.

21. Berry RJ (1977). Reproductive survival and hypoxic protection as a function of radiation ionization density: further studies with murine leukaemia cells irradiated in vitro with mono-energetic accelerated charged particles and assayed in vivo. Radiation Research 70(2) 355-361.

22. Fowler JF (1981), p109 in Nuclear Particles in Cancer Treatment, Medical Physics Handbook, Pubs Adam Hilger with Hospital Physicists Association, Bristol UK

23. A. Perris, P. Pialoglou, A.A., Katsanos \& E.G. Sideris. Biological Effectiveness of Low Energy Protons. I. Survival of Chinese Hamster Cells. Int J Radiat Biol. 1986:1093-1101.

24. Folkard M, Prise KM, Voijnovic B, et al. Inactivation of V79 cells by low-energy protons, deuterons and helium-3 ions. Int J Radiat Biol. 1996;69:729-38.

25. Britten RA, Nazaryan V, Davis LK, Klein SB, Nichiporov D, Mendonca MS et al. Variations in the RBE for cell killing along the depth-dose profile of a modulated proton therapy beam. Radiat Res. 2013 Jan;179(1):21-8.

26. Waligorski MPR, Grzanka L and Korcyl M. The principles of Katz`s cellular track structure radiobiological model. Radiation Protection Dosimetry, 2015 Sep;166(1-4):49-55.

27. Roots R and Okada S. Estimation of Life Times and Diffusion Distances of Radicals Involved in X-Ray-Induced DNA Strand Breaks or Killing of Mammalian Cells. Radiation Research, 64(2): 306-320, 1975.

28. Goodhead DT, Brenner DJ. Estimation of a single property of low LET radiations which correlates with biological effectiveness. Phys Med Biol. 1983 May;28(5):485-92.

29. Charlton DE, Goodhead DT, Wilson WE Paretzke HG (1985). The Deposition of Energy in Small Cylindrical Targets by High LET Radiations. Radiation Protection Dosimetry, 13, $123-125$. 
30. Anderson RM, Stevens DL, Sumption ND, Townsend KM, Goodhead DT, Hill MA (2007). Effect of linear energy transfer (LET) on the complexity of alpha-particle-induced chromosome aberrations in human CD34+ cells. Radiat. Res., 167, 541-550

31. Jones B. Chapter 1. Particle physics for biological interactions in: Practical Radiobiology for Proton Therapy Treatment Planning. Bristol and Philadelphia: Institute of Physics Publishing; 2017.

32. W. Pohlit. Interaction of Energy Depositions and Primary Radiation Lesions Along a Particle Track and Between Particle Tracks Radiation Protection Dosimetry, Volume 13, Issue 1-4, 1 December 1985, Pages 271-273,

33. Lühr A, von Neubeck C, Helmbrecht S, Baumann M, Enghardt W, Krause M. Mødeling in vivo relative biological effectiveness in particle therapy for clinically relevant endpoints. Acta Oncol. 2017 Nov;56(11):1392-1398.

34. Elsässer T, Krämer M, Scholz M, 2008. Accuracy of the local effect model for the prediction of biologic effects of carbon ion beams in vitro and in vivo. Int $\mathrm{J}$ Radiat Oncol Biol Phys. 2008 Jul 1;71(3):866-72

35. Hawkins RB. The relationship between the sensitivity of cells to high-energy photons and the RBE of particle radiation used in radiotherapy. Radiat Res. 2009 Dec;172(6):761-76

36. Guan F, Bronk L, Titt U, Lin SH, Mirkovic D, Kerr MD, et al. Spatial mapping of the biologic effectiveness of scanned particle beams: towards bíologically optimized particle therapy. Sci Rep. 2015 May 18;5:9850. doi: 10.1038/srep09850. 